\title{
Visual and Acoustic Data Analysis for the Bridge Deck Inspection Robotic System
}

\author{
Hung Manh La ${ }^{a}$, Nenad Gucunski ${ }^{b}$, Seong-Hoon Kee ${ }^{c}$ and Luan Nguyen ${ }^{d}$ \\ ${ }^{a}$ Department of Computer Science and Engineering, University of Nevada, Reno, USA \\ ${ }^{b}$ Department of Civil and Environmental Engineering, Rutgers University, Piscataway, USA \\ ${ }^{c}$ Department of Architectural Engineering, Dong-A University, Busan, Korea \\ ${ }^{d}$ Department of Computer Science, Rutgers University, Piscataway, USA \\ E-mail: hung.m.la@ieee.org, gucunski@rci.rutgers.edu, shkee@dau.ac.kr,lvn12@cs.rutgers.edu
}

\begin{abstract}
-
Bridge deck inspection is essential task to monitor the health of the bridges. This paper reports the data collection and analysis for bridge decks based on our novel robotic system which can autonomously and accurately navigate on the bridge. The developed robotic system can lessen the cost and time of the bridge deck data collection and risks of human inspections. The advanced software is developed to allow the robot to collect visual images and conduct nondestructive evaluation (NDE) measurements. The image stitching algorithm to build a whole bridge image from individual images is presented in detail. The impact-echo (IE) and ultrasonic surface waves (USW) data collected by the robot are analyzed to generate the delamination and concrete elastic modulus maps of the deck.
\end{abstract}

Keywords -

Mobile robotic systems, Bridge deck inspection, Image Stitching, Nondestructive evaluation.

\section{Introduction}

The condition of bridges is critical for the safety of the traveling public and economic vitality of the country. There are many bridges through the U.S. that are structurally deficient or functionally obsolete. Condition monitoring and timely implementation of maintenance and rehabilitation procedures are needed to reduce future costs associated with bridge management. Application of nondestructive evaluation (NDE) technologies is one of the effective ways to monitor and predict bridge deterioration. A number of NDE technologies are currently used in bridge deck evaluation, including impactecho (IE), ground penetrating radar (GPR), electrical resistivity (ER), ultrasonic surface waves (USW) testing, visual inspection, etc. [5], [22]. For a comprehensive and accurate condition assessment, data fusion of simultaneous multiple NDE techniques and sensory measurements is desirable. Automated multi-sensor NDE techniques have been proposed to meet the increasing demands for highly-efficient, cost-effective and safety-guaranteed inspection and evaluation [7].

Automated technologies have gained much attention for bridge inspection, maintenance, and rehabilitation. Mobile robotic inspection and maintenance systems are developed for vision based crack detection and maintenance of highways and tunnels [18], [19], [23]. A robotic system for underwater inspection of bridge piers is reported in [3]. An adaptive control algorithm for a bridge-climbing robot is developed [15]. Additionally, robotic systems for steel structured bridges are developed [2], [16], [21]. In one case, a mobile manipulator is used for bridge crack inspection [20]. A bridge inspection system that includes a specially designed car with a robotic mechanism and a control system for automatic crack detection is reported in [11], [12], [17]. Similar systems are reported in [13]-[15] for vision-based automatic crack detection and mapping and [24] to detect cracks on the bridge deck and tunnel. Edge/crack detection algorithms such as Sobel and Laplacian operators are used.

Difference to all of the above mentioned works, our paper focus on the bridge deck data analysis which is collected by our novel robotic system integrated with advanced NDE technologies. The developed data analysis algorithms allows the robot to build the entire bridge deck image and the global mapping of delamination and elastic modulus of the bridge decks. These advanced data analysis algorithms take into account the advantages of the accurate robotic localization and navigation to provide the high-efficient assessments of the bridge decks.

The paper is organized as follows. In the next section, we describe the robotic data collection system and coordinate transformation. In Section III we present the image stitching algorithm and bridge deck viewer/monitoring software. In Section IV, we present 


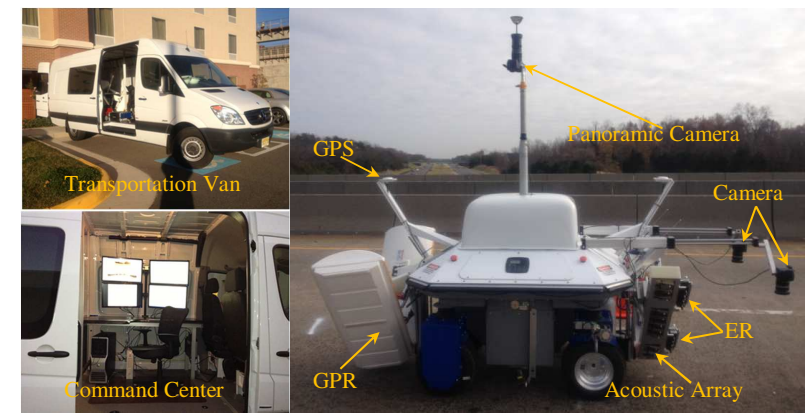

Fig. 1. The bridge robotic inspection system with the command center built in the transportation van.

the IE and USW data collection and analysis. Finally, we provide conclusions from the current work and discuss the future work in Section V.

\section{The bridge robotic inspection system}

\section{A. Data Collection}

The robotic system is integrated with several nondestructive evaluation (NDE) sensors such as Ground Penetrating Radar (GPR), acoustic array consisting of Impact Echo (IE) and Ultrasonic Surface Waves (USW), Electrical Resistivity (ER), and high resolution cameras as shown in Fig. 1. The robot autonomously maneuvers on the bridge based on the advanced localization and navigation algorithm reported in our previous works [8]-[10]. The NDE data collection system is run on two Windows operating computers and communicate with the robot Linux operating computer through the serial communication protocols. The NDE software is developed by utilizing Qt development kit and Cpp to enable the robot to collect and monitor the data simultaneously. The software architecture is designed based on multi-thread programming. The software consists of five slave threads and one master thread. The master thread controls the entire user interface. The slave threads are:

Robot thread which communicates with LinuxWindowsSerial program in the robot computer (Linux / ROS) using RS-232 protocol and sends position information of robot to the user interface;

Acoustic thread which controls the data acquisition of the acoustic device consisting of IW and USW using USB protocol and logs the time series data;

GPR thread which communicates with IDS vendor software using TCP/IP protocol. GPR thread is able to start, stop, and receive stream data from the GPR acquisition device;

Camera thread which uses the Canon SDK protocol to control the camera such as triggering to shoot, changing lighting parameters, and downloading collected images;

Electrical Resistivity (ER) thread which communicates with Resipod sensor using RS-232 protocol and $\log$ s the resipod data.

Overall, the robot thread controls the other threads to trigger and sync the data collection system. During the operation, the robot thread waits for a serial message from robot Linux computer. When the serial message is received, it will be used to command the other NDE thread to perform the data collection. The data flow of the NDE GUI is shown in Fig. 2. The serial message also consists of the robot position and orientation, and number of line inspections and their indices.

\section{B. NDE Coordinate Transformations}

This subsection presents coordinate transformations in the robotic system which allows the NDE data analysis and mapping process. Since the relationship between the GPR, Acoustic, ER coordinates and the robot coordinate are fixed, we just present the transformation from camera frame to robot frame which allows the image stitching and crack mapping process to map from the local image coordinate to the world coordinate.

The system involves four coordinate systems as shown in Fig. 3. They are: image coordinate system $\left(F_{I}\right)$, camera coordinate system $\left(F_{C}\right)$, robot coordinate system $\left(F_{R}\right)$ and world coordinate system $\left(F_{W}\right)$. To transform the image coordinate system $\left(F_{I}\right)$ to the world coordinate system $\left(F_{W}\right)$, we need to implement the sequential transformations: $\left(X_{i m}, Y_{i m}\right) \stackrel{{ }^{I} T_{C}}{\rightarrow}\left(X_{c}, Y_{c}\right) \stackrel{{ }^{C} T_{R}}{\rightarrow}\left(X_{r}, Y_{r}\right) \stackrel{{ }^{R} T_{W}}{\rightarrow}$ $\left(X_{w}, Y_{w}\right)$.

The intrinsic and the extrinsic matrices are obtained once the calibration is finished. The intrinsic matrix consisting of focal length $(f)$, skew value $(s)$ and the origin of image coordinate system $\left(x_{i m}(0), y_{i m}(0)\right)$ is described in Equ. (1).

$$
P=\left[\begin{array}{rrrr}
s f & 0 & x_{i m}(0) & 0 \\
0 & f & y_{i m}(0) & 0 \\
0 & 0 & 1 & 0
\end{array}\right]
$$

The extrinsic matrix consists of rotation and translation parameters as in Equ. (2).

$$
M=\left[\begin{array}{rr}
R & T \\
\mathbf{0} & 1
\end{array}\right]_{4 \times 4}
$$

here $R$ is a $3 \times 3$ rotation matrix which can be defined by the three Euler angles [6], and $T=\left[t_{x}, t_{y}, t_{z}\right]^{T}$ is the translation between two frames.

We have the following transformation from the image 


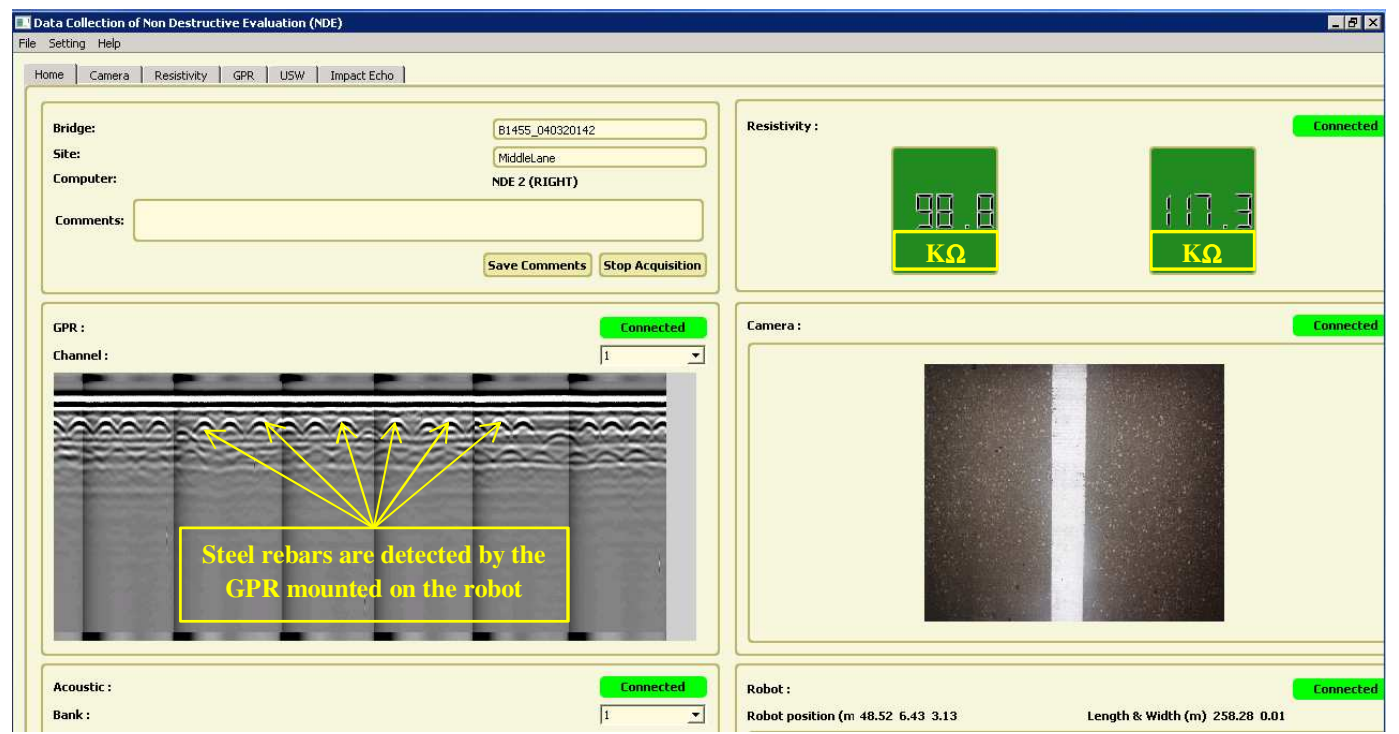

Fig. 2. The GUI for NDE data collection and monitoring of a bridge near Chicago, Illinois, USA.

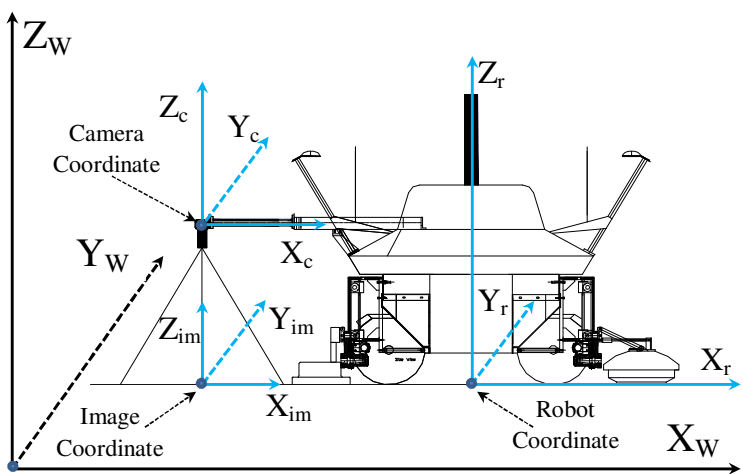

Fig. 3. Coordinate systems in the robotic bridge deck inspection system.

coordinate to the camera coordinate.

$$
\underbrace{\left[\begin{array}{c}
x_{i m} \\
y_{i m} \\
1
\end{array}\right]}_{I}={ }^{I} T_{C} \times \underbrace{\left[\begin{array}{c}
x_{c} \\
y_{c} \\
z_{c} \\
1
\end{array}\right]}_{C},
$$

here ${ }^{I} T_{C}$ is the transformation matrix from the image coordinate to the camera coordinate, and ${ }^{I} T_{C}=P M$. Now we can find the camera coordinate corresponding to the image coordinate using pseudo-inverse as

$$
C=\left({ }^{I} T_{C}^{\prime}{ }^{I} T_{C}\right)^{-1 I} T_{C}^{\prime} I .
$$

here ${ }^{I} T_{C}^{\prime}$ is the transpose of ${ }^{I} T_{C}$.

To find the transformation from $\left(F_{C}\right)$ to $\left(F_{R}\right)$, we use the static relationship between these two coordinates.
Namely, the relationship between the camera and robot coordinate systems is fixed because the camera orientation is fixed (see Fig. 3). Therefore the transformation from $\left(F_{C}\right)$ to $\left(F_{R}\right)$ can be obtained by measuring the physical offset distances between the robot center the camera pose. This transformation can be described as: $\left[\begin{array}{ll}x_{r}^{\text {tran }} & y_{r}^{\text {tran }}\end{array}\right]^{T}=\left[\begin{array}{ll}x_{c} & y_{c}\end{array}\right]^{T}-\left[\begin{array}{ll}\Delta x_{c r} & \Delta y_{c r}\end{array}\right]^{T}$, here $\Delta x_{c r}$ and $\Delta y_{c r}$ are the offset distances between the camera coordinate and the robot coordinate along $x$ and $y$, respectively.

Finally, to find the transformation from $\left(F_{R}\right)$ to $\left(F_{W}\right)$ we use the following relationship:

$$
\left[\begin{array}{c}
x_{w} \\
y_{w} \\
1
\end{array}\right]={ }^{R} T_{W} \times\left[\begin{array}{c}
x_{r}^{\text {tran }} \\
y_{r}^{\text {tran }} \\
1
\end{array}\right],
$$

here the transformation matrix ${ }^{R} T_{W}$ is defined as

$$
{ }^{R} T_{W}=\left[\begin{array}{ccc}
\cos \left(\theta_{r}\right) & -\sin \left(\theta_{r}\right) & x_{r} \\
\sin \left(\theta_{r}\right) & \cos \left(\theta_{r}\right) & y_{r} \\
0 & 0 & 1
\end{array}\right]
$$

where $\left(x_{r}, y_{r}, \theta_{r}\right)$ are the position and heading of the robot obtained by the Extended Kalman Filter (EKF) [10].

\section{Bridge Deck Image Stitching}

\section{A. Bridge Deck Image Stitching}

For the ease of bridge deck inspection and monitoring, we combine taken photos into a single large image as shown in Fig. 4. This is a specific case of the general image stitching problem. In image stitching problem, 

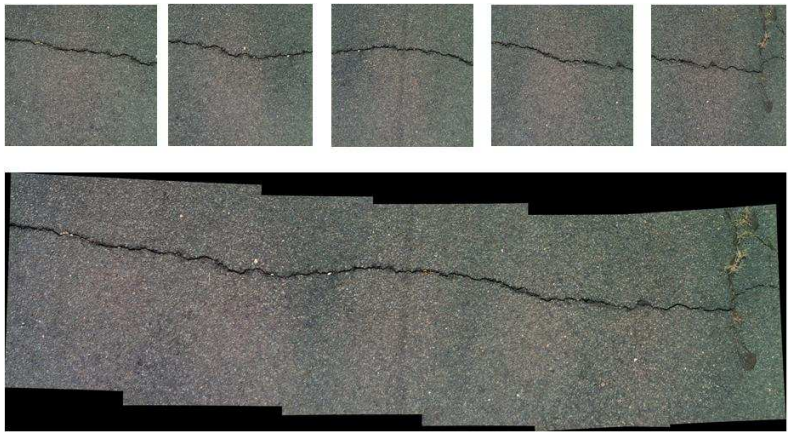

Fig. 4. The result of image stitching from 5 images.

camera motion is unknown and not constrained and intrinsic camera parameters can change between the given images. In our specific problem of bridge deck surface image stitching, we benefit from constraints we know to exist due to the nature of the problem and the setup of the hardware system. We have two identical cameras that simultaneously take images of different but overlapping areas of the bridge. Also the robot's estimated position each time a photo is taken is known with the help of onboard sensor fusion based EKF [10].

\section{1) Motion Estimation}

Based on the constraints imposed by the setup, we estimate the motion as a $2 \mathrm{D}$ rigid motion model; translation on the $x-y$ plane and rotation around $z$ axis. Robot and image coordinate systems can be mapped to each other by -90 degrees rotation. Robot $x$-axis corresponds to negative $y-a x i s$ of image coordinates, robot $y$-axis corresponds to negative $x$-axis of image coordinates (7), and the constant factor resolution is the pixels per meter ratio $\left(R_{i m}\right)$.

$$
\left\{\begin{array}{l}
x_{i m}=-y_{r} R_{i m} \\
y_{i m}=-x_{r} R_{i m}
\end{array}\right.
$$

Sparse feature-matching and image-to-image matching procedures [1], [4] are used to estimate the camera motion incrementally. We pose the problem as a template-matching problem that tries to find the location of the overlapping area of the images inside the other image. This way we perform left-to-right and frame-to-frame matching. Robot motion estimate gives us the rough location of overlapping area for consecutive frames. Rough overlapping area for left-to-right images matching is fixed since the camera locations on the platform are fixed. Knowing the overlapping area, appearance-based template matching can give finer estimation of the camera motion. If the robot motion estimation is not accessible or not accurate enough, overlapping area can be searched over the whole image, which is a more time consuming process.

To reduce the tremendous amount of data to be processed, we resort to multi-resolution pyramidal search method [4], where we search for a larger motion range in lower resolution image and reduce the possible motion range for higher resolution image. Due to possible large illumination and reflection changes between different frames, we use image comparison method Normalized Correlation Coefficient (8) that is less illumination independent. In Equ. (8) correlation coefficient for each location $x, y$ is denoted by $R(x, y)$, where search image region is $I$, template image that is searched is $T$ and normalized versions of them are $I^{\prime}$ and $T^{\prime}$ respectively. We compare the grayscale versions of the images to get rid of any white-balance effects in different images.

$$
\left\{\begin{array}{l}
R(x, y)=\frac{\sum_{x^{\prime}, y^{\prime}}\left[T^{\prime}\left(x^{\prime}, y^{\prime}\right) I^{\prime}\left(x+x^{\prime}, y+y^{\prime}\right)\right]}{\sqrt{\sum_{x^{\prime}, y^{\prime}} T^{\prime}\left(x^{\prime}, y^{\prime}\right)^{2} \sum_{x^{\prime}, y^{\prime} I^{\prime}\left(x+x^{\prime}, y+y^{\prime}\right)^{2}}}} \\
I^{\prime}\left(x+x^{\prime}, y+y^{\prime}\right)=I\left(x+x^{\prime}, y+y^{\prime}\right)- \\
\frac{\sum_{x^{\prime \prime}, y^{\prime \prime}} I\left(x+x^{\prime \prime}, y+y^{\prime \prime}\right)}{w \cdot h} \\
T^{\prime}\left(x^{\prime}, y^{\prime}\right)=T\left(x^{\prime}, y^{\prime}\right)-\frac{\sum_{x^{\prime \prime}, y^{\prime \prime} T\left(x^{\prime \prime}, y^{\prime \prime}\right)}^{w \cdot h}}{}
\end{array}\right.
$$

here, $w$ and $h$ are the width and height of the image $I$, respectively.

\section{2) Exposure Compensation and Blending}

Exposure compensation step obtains the most blending exposures for each image by selecting the suitable brightness ratio of overlapping area between images. Then when combining existing image and the new arrived image, we are performing an image-blending step to remove the shadows in the image (9). If the new arriving pixel is considerably brighter than the existing pixel in the same location, we replace the pixel with the new one. A threshold value of 0.7 is used for th to indicate being considerable is brighter than corresponding pixel.

$$
I(x, y)=f(x)\left\{\begin{array}{l}
I_{2}(x, y), I_{2}(x, y) * t h>I_{1}(x, y) \\
I_{1}(x, y), \text { else }
\end{array}\right.
$$

Figure 5 shows the image stitching result. There are 200 images collected by the two front surface cameras.

\section{B. Bridge Deck Monitor}

The bridge deck viewer (BDV) software (see Fig. 6) is developed using Java language to support the bridge engineer to monitor the bridge decks in an efficient way. The stitched images are first loaded and then calibrated to map to the bridge coordinate as Fig. 7. The BDV 


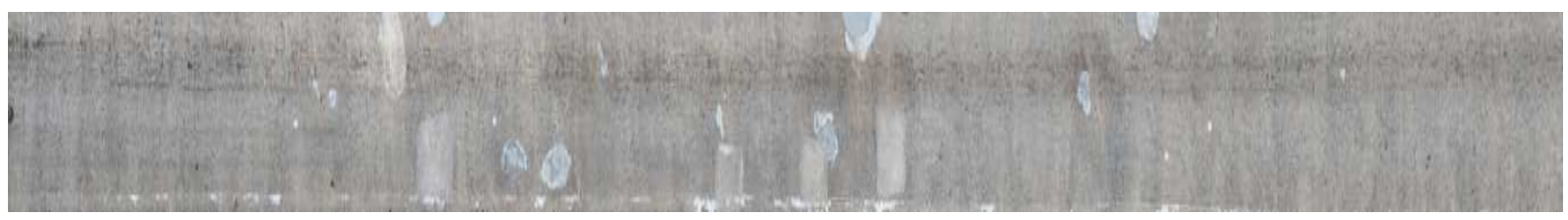

Fig. 5. The result of image stitching from 200 images collected by two cameras.

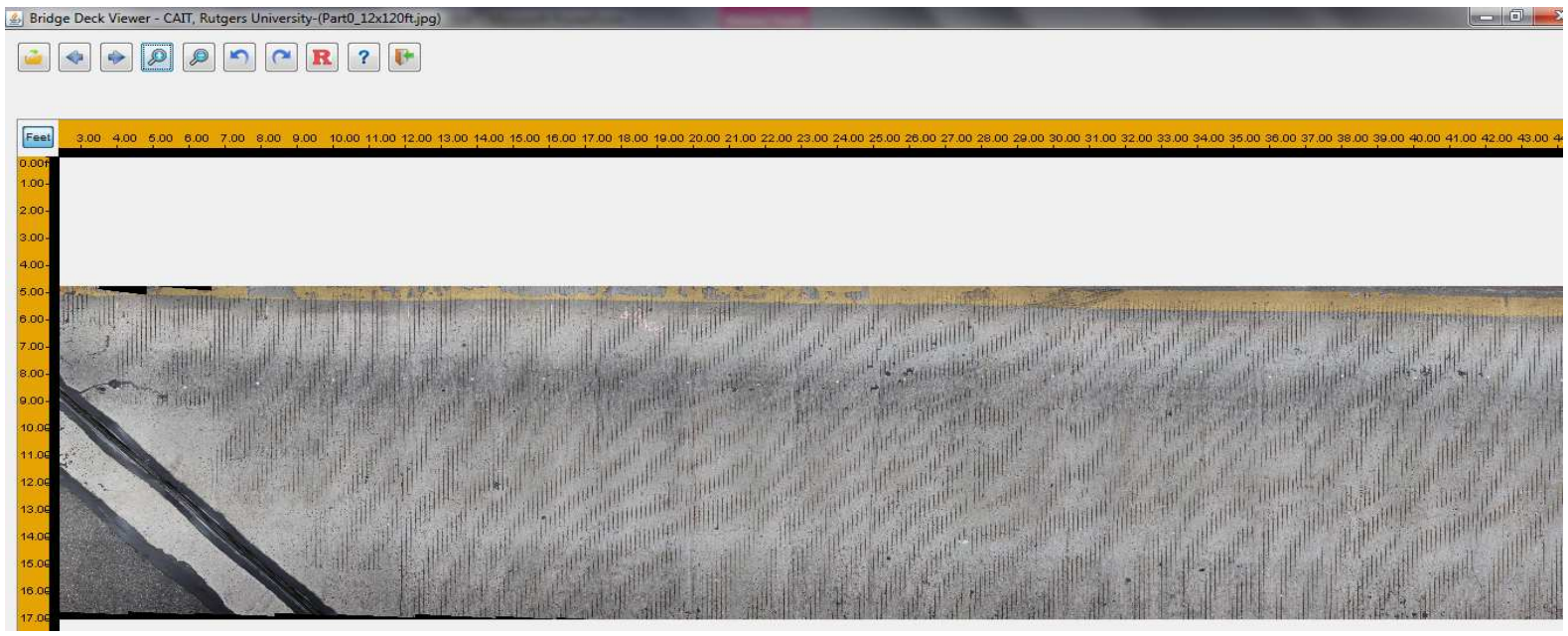

Fig. 7. One of New Jersey bridges is loaded and calibrated by the BDV software.

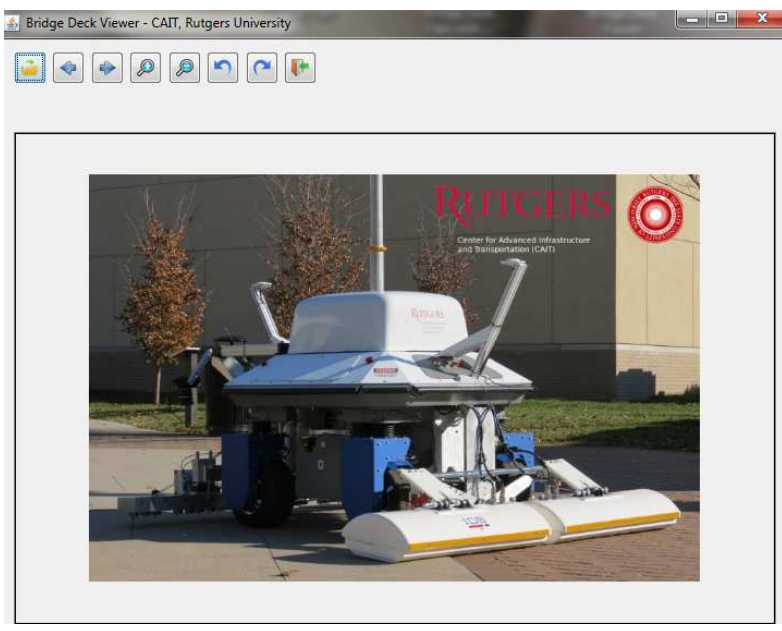

Fig. 6. The interface of the bridge deck monitor software.

software can find the crack locations on the surface of bridge in the viewing image and allows to mark them for the next view or any purpose by left mouse click on that locations. The BDV also shows the notification about the position of the cracks. As can be seen in Fig. 8 , the flags appear at the crack locations corresponding

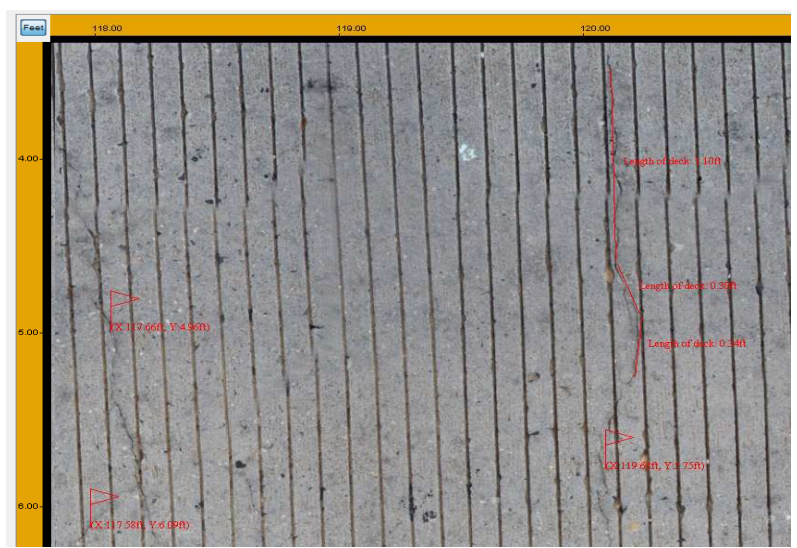

Fig. 8. Zoom-in at some crack locations of a bridge in New Jersey.

with coordinates $(\mathrm{x}, \mathrm{y})$ on the bridge deck.

Additionally, the BDV software allows to measure the distance of crack on the deck by right mouse click on the starting point and drag the hold right mouse to the last point. A line that connects the starting point and ending point appears to show the length of the crack as shown in Fig. 8. 


\section{IE and USW Data Collection and Anal- ysis}

This section presents impact-echo (IE) and ultrasonic surface waves (USW) data collection and anlysis. The robot is equipped with two acoustic arrays, and each array can produce $8 \mathrm{IE}$ and 6 USW data set as shown in Fig. 9. These raw data sets are collected by the robot at every two feet $(60 \mathrm{~cm})$ on the bridge deck.

\section{A. Impact-Echo (IE) Data Analysis}

Impact-Echo (IE) is an elastic-wave based method to identify and characterize delaminations in concrete structures. This method uses the transient vibration response of a plate-like structure subjected to a mechanical impact. The mechanical impact generates body waves (P-waves or longitudinal waves and S-waves or transverse waves), and surface-guided waves (e.g. Lamb and Rayleigh surface waves) that propagate in the plate. The multiple-reflected and mode-converted body waves eventually construct infinite sets of vibration resonance modes within the plate. In practice, the transient time response of the solid structure is commonly measured with a contact sensor (e.g., a displacement sensor or accelerometer) coupled to the surface close to the impact source. The fast Fourier transform (amplitude spectrum) of the measured transient time-signal will show maxima (peaks) at certain frequencies, which represent particular resonance modes as show in Fig. 10.

There are different ways of interpreting the severity of the delamination in a concrete deck with the IE method. One of the ways used in this study is shown in Fig. 11. A test point is described as solid if the dominant frequency corresponds to the thickness stretch modes (Lamb waves) family. In that case, the frequency of the fundamental thickness stretch mode (the zerogroup-velocity frequency of the first symmetric $\left(S_{1}\right)$ Lamb mode, or also called the IE frequency $\left(f_{I E}\right)$. The frequency can be related to the thickness of a plate $H$ for a known $P$-wave velocity $C_{p}$ of concrete by

$$
H=\frac{\beta_{1} C_{p}}{f_{I E}}
$$

where $\beta_{1}$ is a correction factor that depends on Poisson's ratio of concrete, ranging from 0.945 to 0.957 for the normal range of concrete. A delaminated point in the deck will theoretically demonstrate a shift in the thickness stretch mode toward higher values because the wave reflections occur at shallower depths. Depending on the extent and continuity of the delamination, the partitioning of the wave energy reflected from the bottom of the deck and the delamination may vary. The initial or incipient delamination, described as occasional

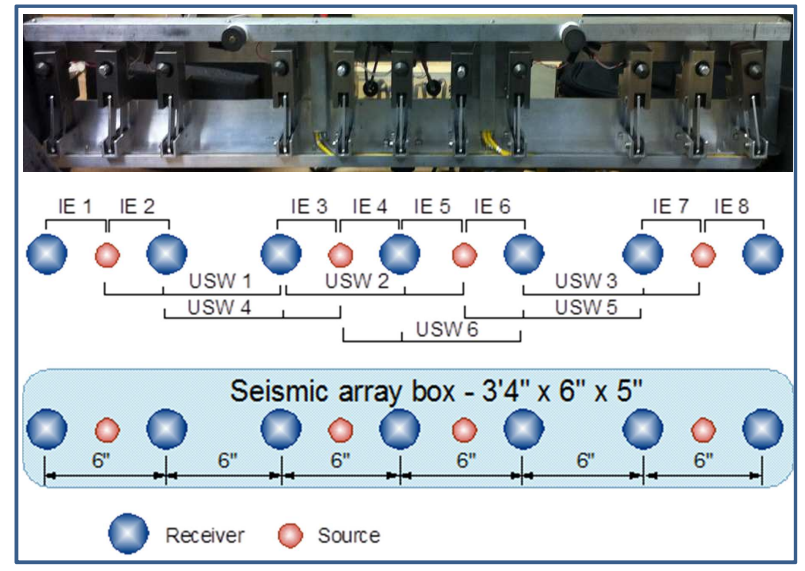

Fig. 9. Acoustic/seismic array sensor is developed and integrated with the robot to collect IE and USW data.

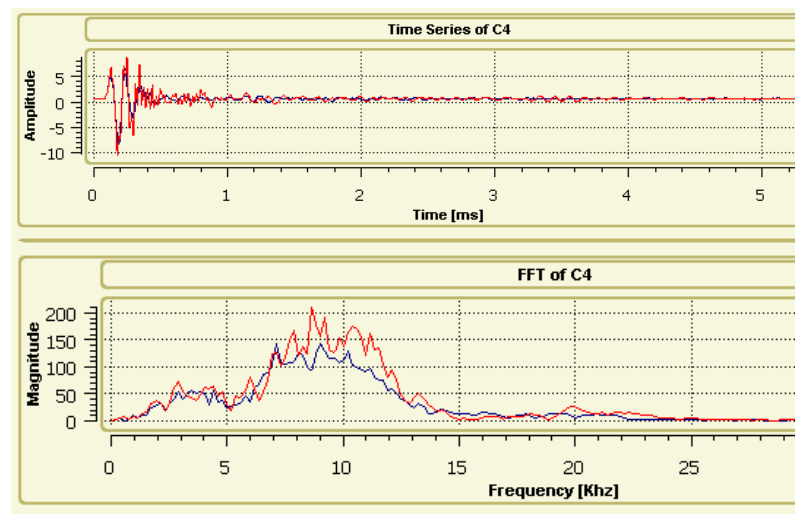

Fig. 10. (a) IE raw data of one chanel, C4, in the acoustic array in time domain, and (b) After using Fast Fourier Transform (FFT) in frequency domain.

separation within the depth of the slab, can be identified through the presence of dominant frequencies associated with the thickness stretch modes from both the bottom of the deck and the delamination. Progressed delamination is characterized by a single peak at a frequency corresponding to the depth of the delamination. Finally, in case of wide or shallow delaminations, the dominant response of the deck to an impact is characterized by a low frequency response of flexural-mode oscillations of the upper delaminated portion of the deck.

\section{B. Ultrasonic Surface Waves (USW) Data Analysis}

The ultrasonic surface waves (USW) technique is an offshoot of the spectral analysis of surface waves (SASW) method used to evaluate material properties 


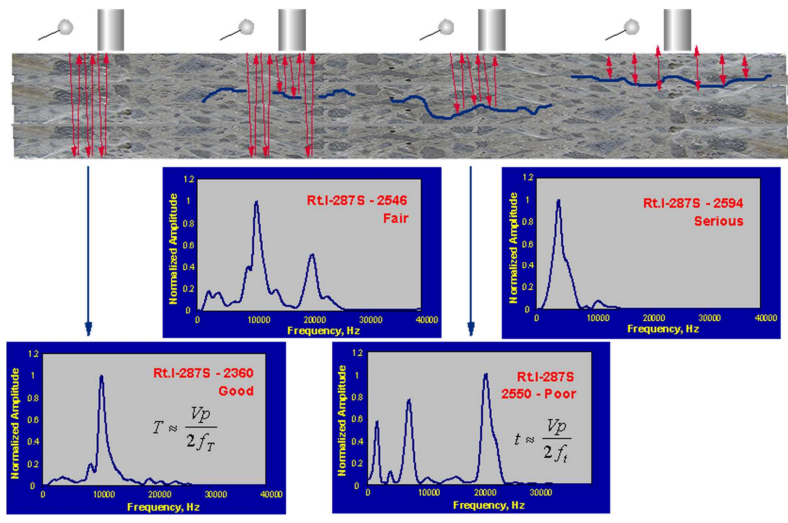

Fig. 11. Grades for various degrees of deck delamination for IE method.

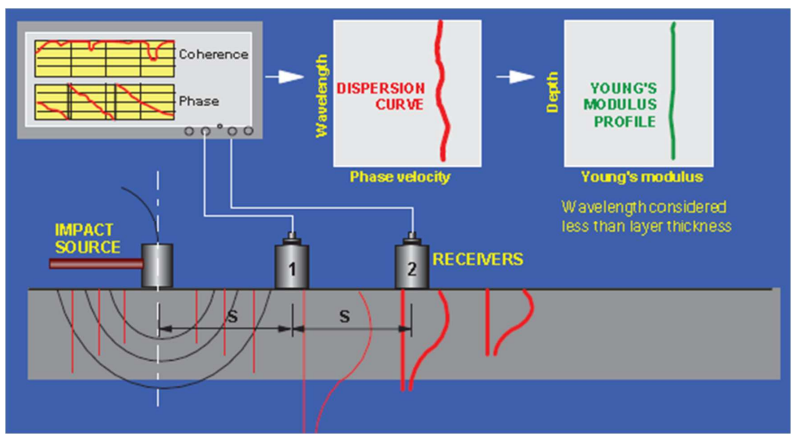

Fig. 12. Schematic of evaluation of a layer modulus by SASW (USW) method.

(elastic moduli) in the near-surface zone. The SASW uses the phenomenon of surface wave dispersion (i.e., velocity of propagation as a function of frequency and wave length, in layered systems to obtain the information about layer thickness and elastic moduli) as shown in Fig. 12. A SASW test consists of recording the response of the deck, at two receiver locations, to an impact on the surface of the deck. The surface wave velocity can be obtained by measuring the phase difference $\Delta \phi$ between two different sensors (sensor 1 and sensor 2 ) as follows,

$$
C=2 \pi f \frac{d}{\Delta \phi}
$$

where $f$ is frequency, $d$ is distance between two sensors. The USW test is identical to the SASW, except that the frequency range of interest is limited to a narrow highfrequency range in which the surface wave penetration depth does not exceed the thickness of the tested object. In cases of relatively homogeneous materials, the velocity of the surface waves does not vary significantly with frequency. The surface wave velocity can be precisely related to the material modulus, or concrete modulus in the case of bridge decks, using either the measured or assumed mass density, or Poisson's ratio of the material. In the case of a sound and homogenous deck, the velocity of the surface waves will show little variability. An average velocity is used to correlate it to the concrete modulus. Significant variation in the phase velocity will be an indication of the presence of a delamination or other anomaly.

The IE condition map is presented in Fig. 13-top. The test regions classified as serious condition (red color) are interpreted as likely delaminated areas on the concrete deck. The USW condition map is presented in Fig. 13-bottom. The USW map provides the condition assessment and quality of concrete through measuring concrete modulus. The presented modulus plot indicates that zones of very low moduli provide a good match with delaminated zones identified by other methods.

\section{Acknowledgment}

This work was supported by the Federal Highway Administration's Long Term Bridge Performance (LTBP) Program. The authors would like to thank Profs. Basily Basily, Kristin Dana, Jingang Yi and Ali Maher of Rutgers University for their support for the project development. The authors are also grateful to Ronny Lim, Turgay Senlet, Hooman Parvardeh, Kenneth Lee, Prateek Prasanna, Yizhai Zhang, Moiz Ezzy and Fei Liu of Rutgers University for their help during the system development and field testing.

\section{Conclusions and Future Work}

The bridge deck data collection and analysis has been reported in this paper. Several challenging problems of data collection software, image stitching, IE and USW analysis have been tackled. The image stitching algorithm allowed to generate a very high resolution image of the whole bridge deck, and the bridge viewer software allows to calibrate the stitched image to the bridge coordinate. The delamination and elastic modulus maps were built based on IE and USW data collected by the robot to provide easy evaluation and condition monitoring of bridge decks. Extensive testings and deployments of the proposed system on a number of bridges proved the efficiency of the new approach for bridge deck inspection and evaluation.

In the future work we will include development of a fusion algorithm for the NDE sensor and camera data for a more comprehensive and intuitive bridge deck condition assessment data presentation. 

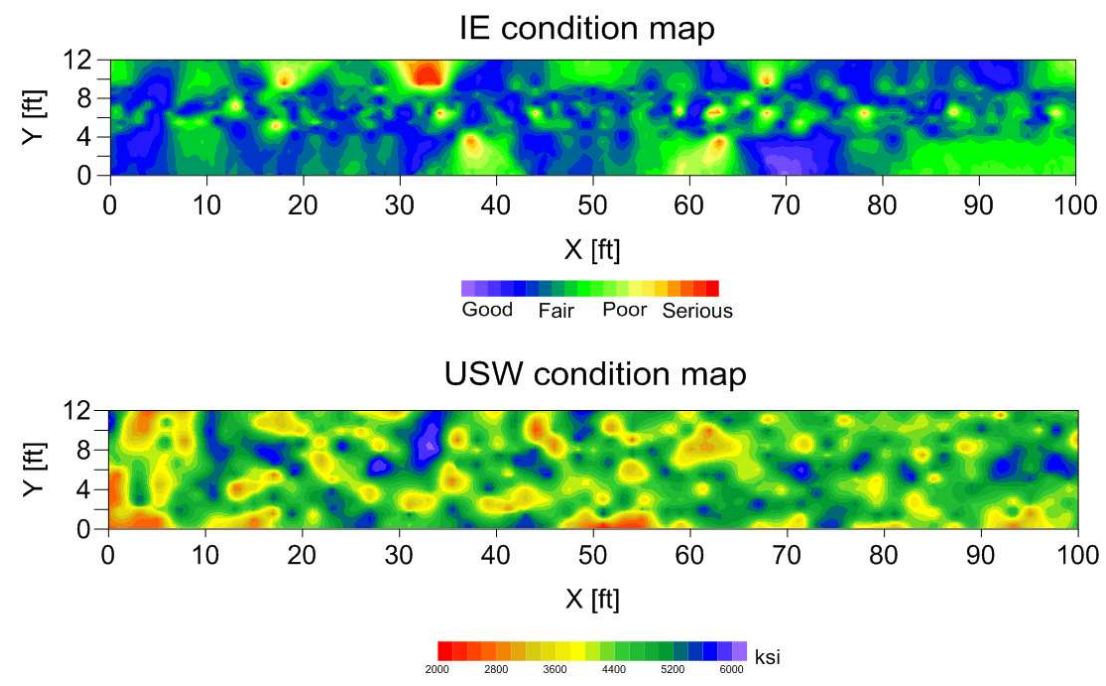

Fig. 13. The impact-echo (IE) and ultrasonic surface waves (USW) condition maps of a bridge deck in Illinois, USA, based on data collected by the developed robot system. The robot covers a half of the bridge with two scans (6ft width/scan) and 100ft along within 25 minutes.

\section{References}

[1] M. Brown and D. G. Lowe. Automatic panoramic image stitching using invariant features. Int. J. of Computer Vision, 74(1):59-73, 2007.

[2] Kyeong Ho Cho, Ho Moon Kim, Young Hoon Jin, Fengyi Liu, Hyungpil Moon, Ja Choon Koo, and Hyouk Ryeol Choi. Inspection robot for hanger cable of suspension bridge: Mechanism design and analysis. IEEE/ASME Trans. on Mechatronics, 18(6):1665-1674, Dec 2013.

[3] J. E. DeVault. Robotic system for underwater inspection of bridge piers. IEEE Instrumentation Measurement Magazine, 3(3):32-37, Sep 2000.

[4] D. A. Forsyth and J. Ponce. Computer Vision: A Modern Approach. Prentice Hall, Upper Saddle River, NJ, 2003.

[5] N. Gucunski, F. Romero, S. Kruschwitz, R. Feldmann, A. AbuHawash, and M. Dunn. Multiple complementary nondestructive evaluation technologies for condition assessment of concrete bridge decks. Transp. Res. Rec., 2201:34-44, 2010.

[6] J. Heikkila. Geometric camera calibration using circular control points. IEEE Trans. Pattern Anal. Machine Intell., 22(10):10661077, 2000.

[7] D. Huston, J. Cui, D. Burns, and D. Hurley. Concrete bridge deck condition assessment with automated multisensor techniques. Struct. Infrastruct. Eng., 7(7-8):613-623, 2011.

[8] H. M. La, N. Gucunski, S. H. Kee, J. Yi, T. Senlet, and L. Nguyen. Autonomous robotic system for bridge deck data collection and analysis. In the Proc. of the IEEE/RSJ Inter. Conf. on Intelligent Robots and Systems (IROS), Sept. 2014.

[9] H. M. La, R. Lim, B. Basily, N. Gucunski, J. Yi, A. Maher, F. Romero, and H. Parvardeh. Autonomous robotic system for high-efficiency non-destructive bridge deck inspection and evaluation. In Proc. IEEE Conf. Automat. Sci. Eng., pages 10651070, Madison, WI, 2013.

[10] H. M. La, R. S. Lim, B. B. Basily, N. Gucunski, J. Yi, A. Maher, F. A. Romero, and H. Parvardeh. Mechatronic systems design for an autonomous robotic system for high-efficiency bridge deck inspection and evaluation. IEEE/ASME Trans. Mechatronics, 18(6):1655-1664, 2013.

[11] J. H. Lee, J. M. Lee, J. W. Park, and Y. S. Moon. Efficient algorithms for automatic detection of cracks on a concrete bridge.
In Proc. 23rd Int. Tech. Conf. Circ./Syst., Comp. Communicat., pages 1213-1216, Yamaguchi, Japan, 2008.

[12] J. H. Lee, J.M. Lee, H. J. Kim, and Y.S. Moon. Machine vision system for automatic inspection of bridges. In Cong. Image Sig. Proc., volume 3, pages 363-366, Sanya, China, 2008.

[13] R. S. Lim, H. M. La, Z. Shan, and W. Sheng. Developing a crack inspection robot for bridge maintenance. In Proc. IEEE Int. Conf. Robot. Autom., pages 6288-6293, Shanghai, China, 2011.

[14] R. S. Lim, H. M. La, and W. Sheng. A robotic crack inspection and mapping system for bridge deck maintenance. IEEE Trans. on Automat. Sci. and Eng., 11(2):367-378, Apr. 2014.

[15] Q. Liu and Y. Liu. An approach for auto bridge inspection based on climbing robot. In IEEE Inter. Conf. on Robotics and Biomimetics, pages 2581-2586, Dec 2013.

[16] A. Mazumdar and H. H. Asada. Mag-foot: A steel bridge inspection robot. In IEEE/RSJ Inter. Conf. on Intelligent Robots and Systems, pages 1691-1696, Oct 2009.

[17] J. K. Oh, G. Jang, S. Oh, J. H. Lee, B. J. Yi, Y. S. Moon, J. S. Lee, and Y. Choi. Bridge inspection robot system with machine vision. Automat. Constr., 18:929-941, 2009.

[18] S. A. Velinsky. Heavy vehicle system for automated pavement crack sealing. Int. J. Veh. Design, 1(1):114-128, 1993.

[19] S. J. Lorenc and B. E. Handlon and L. E. Bernold. Development of a robotic bridge maintenance system. Automat. Constr., 9:251258, 2000.

[20] P. C. Tung, Y. R. Hwang, and M. C. Wu. The development of a mobile manipulator imaging system for bridge crack inspection. Automat. Constr., 11:717-729, 2002.

[21] X. Wang and F. Xu. Conceptual design and initial experiments on cable inspection robotic system. In IEEE Inter. Conf. on Mechatronics and Automation, pages 3628-3633, Aug 2007.

[22] Z. W. Wang, M. Zhou, G. G. Slabaugh, J. Zhai, and T. Fang. Automatic detection of bridge deck condition from ground penetrating radar images. IEEE Trans. Automat. Sci. Eng., 8(3):633640, 2011.

[23] S. N. Yu, J. H. Jang, and C. S. Han. Auto inspection system using a mobile robot for detecting concrete cracks in a tunnel. Automat. Constr., 16:255-261, 2007.

[24] S.-N. Yu, J.-H. Jang, and C.-S. Han. Auto inspection system using a mobile robot for detecting concrete cracks in a tunnel. Automat. Constr., 16:255-261, 2007. 